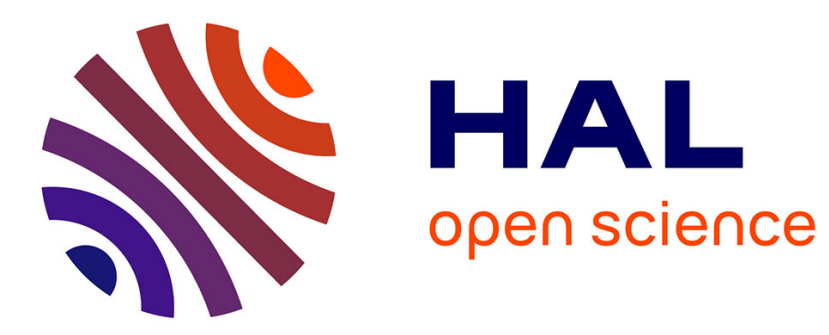

\title{
Concentrated Winsor microemulsions: a small angle X-ray scattering study
}

\author{
L. Auvray, J.-P. Cotton, R. Ober, C. Taupin
}

\section{To cite this version:}

L. Auvray, J.-P. Cotton, R. Ober, C. Taupin. Concentrated Winsor microemulsions: a small angle X-ray scattering study. Journal de Physique, 1984, 45 (5), pp.913-928. 10.1051/jphys:01984004505091300 . jpa-00209825

\section{HAL Id: jpa-00209825 https://hal.science/jpa-00209825}

Submitted on 1 Jan 1984

HAL is a multi-disciplinary open access archive for the deposit and dissemination of scientific research documents, whether they are published or not. The documents may come from teaching and research institutions in France or abroad, or from public or private research centers.
L'archive ouverte pluridisciplinaire HAL, est destinée au dépôt et à la diffusion de documents scientifiques de niveau recherche, publiés ou non, émanant des établissements d'enseignement et de recherche français ou étrangers, des laboratoires publics ou privés. 
Classification

Physics Abstracts

$61.10-61.25-82.70$

\title{
Concentrated Winsor microemulsions : a small angle X-ray scattering study
}

\author{
L. Auvray $\left({ }^{1,2}\right)$, J.-P. Cotton $\left({ }^{2}\right)$, R. Ober $\left({ }^{1}\right)$ and C. Taupin $\left({ }^{1}\right)$ \\ ( $\left.{ }^{1}\right)$ Laboratoire de Physique de la Matière Condensée, \\ Collège de France, 11, place Marcelin-Berthelot, 75231 Paris Cedex 05, France \\ $\left({ }^{2}\right)$ Laboratoire Léon Brillouin, C.E.A.-C.E.N. Saclay, 91191 Gif-sur-Yvette Cedex, France
}

(Reçu le 11 août 1983, révisé le 18 novembre, accepté le 6 janvier 1984)

Résumé. - Nous avons étudié par diffusion centrale des rayons $\mathrm{X}$ la structure de microémulsions de Winsor dont les proportions de saumure et d'huile (toluène) sont comparables et grandes devant les proportions de tensioactif (sodium dodécyl sulfate, $\mathrm{SdS}$ ) et cotensioactif (butanol 1). Trois systèmes ont été fabriqués. Dans le premier (déjà trè̀s étudié) les microémulsions sont en équilibre avec une phase eau et/ou huile en excès, la composition des microémulsions varie en fonction de la salinité. Les deux autres systèmes sont des monophases faites avec une saumure de salinité $6,5 \%$. Dans le second système, nous faisons varier la fraction volumique en huile, $\phi_{0}$, de 20 à $90 \%$ à concentration constante en tensioactif $\left(5 \times 10^{-2} \mathrm{~g} / \mathrm{ml}\right)$. Dans le troisième, nous avons choisi les proportions des constituants de telle sarte que la quantité $\frac{\phi_{0} \phi_{\mathrm{w}}}{c_{\mathrm{s}}}\left(\phi_{\mathrm{w}}\right.$ : fraction volumique en saumure, $c_{\mathrm{s}}$ : concentration de $\mathrm{SdS}$ ) reste constante quand $\phi_{0}$ varie de 20 à $80 \%$. On fixe ainsi la longueur caractéristique théorique des microémulsions, selon l'expression $\xi=\frac{6 \phi_{0} \phi_{\mathrm{w}}}{c_{\mathrm{S}} \Sigma}$ prédite par Talmon-Prager et de Gennes ( $\Sigma$ est la surface par tête polaire de tensioactif).

1) La contribution des têtes polaires au contraste et à l'intensité diffusée aux grands angles dans le domaine asymptotique est substantielle. Nous en déduisons une preuve directe de l'existence d'un film interfacial de savon séparant les parties eau et huile de la microémulsion et une méthode de mesure de la surface par tête polaire $\left(\Sigma \approx 60 \AA^{2}\right)$;

2) Nous mesurons la taille caractéristique des microémulsions $\xi$ en fonction de $\phi_{0}$ et $c_{\mathrm{s}}$. Les différents balayages suggèrent que, pour les systèmes de Winsor, à partir du point d'inversion $\left(\phi_{0}=0,5\right)$, quand la proportion d'eau ou de toluène augmente, on passe progressivement d'une structure aléatoire bicontinue d'eau et d'huile $\left(0,3<\phi_{0}<0,7, \xi \sim \frac{\phi_{0} \phi_{\mathrm{w}}}{c_{\mathrm{s}} \Sigma}\right)$ à une dispersion faite de gouttelettes distinctes d'eau dans l'huile ou d'huile dans l'eau $\left(\phi_{0}<0,3\right.$ ou $\phi_{0}>0,7, \xi \sim \frac{\phi_{0}}{c_{\mathrm{s}} \Sigma}$ ou $\left.\frac{\phi_{\mathrm{w}}}{c_{\mathrm{s}} \Sigma}\right)$;

3) Nous observons aux très petits angles $\left(q \sim 10^{-2} \AA^{-1}\right)$ des effets de corrélations ou d'interactions non prédits par les modèles existants de microémulsions bicontinues.

Abstract. - By small angle X-ray scattering, we have studied the structure of Winsor microemulsions, with brine and oil (toluene) proportions comparable and much larger than the surfactant (sodium dodecyl sulfate, SdS) and cosurfactant (butanol 1) proportions. Three systems were made. In the first (already studied in detail), the microemulsions are in equilibrium with an oil and/or water phase in excess, the microemulsion composition varies as a fonction of the brine salinity. The two other systems are monophases made with a brine of salinity $6.5 \%$. In the second system, we vary the oil volume fraction $\phi_{0}$ from 20 to $90 \%$ at constant $\operatorname{SdS}$ concentration $\left(5 \times 10^{-2} \mathrm{~g} / \mathrm{ml}\right)$. In the third, we chose the component proportions such that the quantity $\frac{\phi_{0} \phi_{\mathrm{w}}}{c_{\mathrm{s}}}\left(\phi_{\mathrm{w}}\right.$ : brine volume fraction, $c_{\mathrm{s}}$ : SdS concentration) remains constant as $\phi_{0}$ varies from 20 to $80 \%$. Thus one fixes the theoretical microemulsion characteristic length according to the expression $\xi=\frac{6 \phi_{0} \phi_{\mathrm{w}}}{c_{\mathrm{S}} \Sigma}$ predicted by Talmon-Prager and de Gennes $(\Sigma$ is the area per SdS polar head).

1) The SdS polar head contribution to the contrast and to the intensity scattered at large angle in the asymptotic domain is large. From this, we deduce a direct proof of the existence of a soap film between the oil and water parts of the microemulsion and also a method of measurement of the area per polar head $\left(\Sigma \approx 60 \AA^{2}\right)$;

2) We measure the characteristic microemulsion size as a function of $c_{\mathrm{S}}$ and $\phi_{0}$. The various scans suggest that, for Winsor microemulsions, starting from the inversion point $\left(\phi_{0}=0.5\right)$ and increasing the water or oil proportion, 
a progressive change occurs from a random bicontinuous water and oil structure $\left(0.3<\phi_{0}<0.7, \xi \sim \frac{\phi_{0} \phi_{\mathrm{w}}}{c_{\mathrm{S}} \Sigma}\right)$ to a dispersion made of distinct oil in water or water in oil droplets $\left(\phi_{0}<0.3\right.$ or $\phi_{0}>0.7, \xi \sim \frac{\phi_{0}}{c_{\mathrm{S}} \Sigma}$ or $\left.\frac{\phi_{\mathrm{w}}}{c_{\mathrm{S}} \Sigma}\right)$;

3) At very small angles $\left(q \sim 10^{-2^{-}} \AA^{-1}\right)$, we observe correlations or interaction effects not predicted by the existing bicontinuous microemulsion models.

\section{Introduction.}

Microemulsions are fluid, transparent, isotropic mixtures of water (or brine) and hydrocarbon (« oil ») stabilized by amphiphilic substances (a surfactant and often a cosurfactant : e.g. a short alcohol) [1]. They exist as single phase or coexist with either an excess of water, or an excess of oil (Winsor II phases), or both (Winsor III phases) [2], (sometimes they also coexist with an oil-water-amphiphiles liquid cristalline phase [3]).

With lipophilic surfactants, one solubilizes water in oil; with hydrophilic surfactants, it is the reverse. Depending on the choice and quantities of surfactant and cosurfactant, and depending on the temperature and salinity, the oil and water proportions in a microemulsion can be practically fixed at will.

At low water (or oil) concentration, the structure of these phases is precisely known : the microemulsion is a dispersion of water in oil (or oil in water) spherical droplets (the radius is $\sim 100 \AA$ ); the oilwater interface is saturated by a dense mixed film of surfactant and cosurfactant.

When the oil and water proportions are comparable (this corresponds to the "inversion zone " of the phase diagram) the structure question becomes more puzzling :

i) In certain cases, well defined spheres still exist : models of hard spheres in interaction successfully interpret the neutron and light scattering data [4]. This type of microemulsions has been particularly studied along dilution-paths in the phase-diagram [5] : for a water in oil microemulsion, the molar ratio water/soap, the composition of the continuous oilalcohol phase and the structure of the water droplets are kept constant as the water-concentration is increased until an upper bound is reached. This bound corresponds either to a solubility limit or to an oil in water microemulsion (inversion). It often coincides with the random sphere-close-packing concentration [5].

ii) In some other cases, the oil or water objects are less well defined : sphere aggregates are evidenced [6].

iii) Finally it may happen that there are no longer any defined objects, but only a bicontinuous random partition of oil and water [7]. This would explain that :

- In some phase diagrams, one can pass continuously from an oil in water to a water in oil structure.
- Non dilutable microemulsions exist (in particular Winsor III microemulsions).

Recently de Gennes and one of us have interpreted part of these various features by considering the curvature elasticity of the interfacial film [8] :

When the film exhibits a strong spontaneous curvature due to a strong dissymmetry between the polar and aliphatic part of the film, well defined objects (oil in water or water in oil) are preferred.

When there is no preferred curvature but a large rigidity of the film, the system tends to build well organized lamellar phases.

An interesting case corresponds to a weak spontaneous curvature and a flexible interfacial film. In this case, a bicontinuous random structure exists at sufficiently high water and oil concentrations. The characteristic size of this structure is the persistence length of the film $\xi_{k}$ (the interfacial film is rigid and flat at scales smaller than $\xi_{k}$ and wrinkled at larger scales) : the volume is divided into domains of size $\xi_{k}$, these domains being randomly filled either by oil or water.

This structure model, derived from the TalmonPrager model [9] also allows an initial discussion of the Winsor type phase equilibria to be given in terms of the surface energy, the film entropy and the curvature energy [10]. The main results are' :

i) the model only generates Winsor II systems

ii) the compositions of the phases in equilibria fall on the line :

$$
\xi_{k} \simeq \frac{6 \phi_{\mathrm{w}} \phi_{0}}{c_{\mathrm{s}} \Sigma}
$$

where $c_{\mathbf{S}}$ is the surfactant concentration (in molecules number per $\AA^{3}$ ), $\Sigma$ is the area per surfactant polar head (in $\AA^{2}$ ), $\phi_{0}$ and $\phi_{\mathrm{w}}$ are respectively the oil and water volume fractions in the phases.

The Winsor microemulsions have been much studied because their solubilization capabilities are high and the interfacial tension between the microemulsion phase and the phase(s) in excess are ultralow $\left(\sim 10^{-3}\right.$ dyne $\left./ \mathrm{cm}\right)$ [11]. But their structures have been less studied and we do not know yet if they enter in the above framework :

- Their phase behaviour depends practically on numerous parameters (salinity, alcohol, temperature) whose rôles are poorly identified.

- In Winsor III system, Van der Waals interactions possibly have to be taken into account [12]. 
- The critical behaviour often observed at the transition Winsor II-Winsor III when one of the parameters is varied, still raises problems.

In order to test in these microemulsions the existence of a bicontinuous structure and the model prediction (1), we have studied their structure by small angle $\mathrm{X}$-ray scattering.

The microemulsion are made of brine, toluene, sodium dodecyl sulfate (SdS) and butanol 1.

We obtained three systems :

The first one (A) has been studied in detail [13-15]. Starting from a mixture whose proportions (by weight) are : brine $47.95 \%$; toluene $46.35 \%$; SdS $1.95 \%$; butanol $3.75 \%$ and varying the salinity of brine ( $S=\mathrm{NaCl}$ weight-percentage of brine), one obtains a series of Winsor phases :

At low salinity $(S<5.5 \%$ ), an oil in water microemulsion coexists with nearly pure toluene; at high salinity $(S>7.5 \%)$ the rôle of oil and water are inverted; in between Winsor III microemulsion coexists with brine and toluene excesses.

In this system, the composition of the microemulsion phases is not controlled. Thus we have also worked on monophasic microemulsions (called B and C) located in the same region of the phase diagram.

The amount of SdS, toluene, and brine was a priori chosen. It was then examined if a given mixture could be titrated by butanol to form a monophasic microemulsion.

This procedure has allowed us :

i) To perform a toluene volume fraction $\left(\phi_{0}\right)$ scan (from $\phi_{0}=20 \%$ to $90 \%$ ) at fixed salinity $(S=6.5 \%)$ and approximatively constant $S \mathrm{~d} S$ concentration (system B). Thus one separates as much as possible the effects of salt (which seems to modify the curvature of the interfacial film) and the effects of butanol.

ii) To make a $\phi_{0}$-scan $\left(0.2<\phi_{0}<0.8\right)$ at $\xi_{k}$ (expressed by formula (1)) approximatively constant (system C).

\section{Experiment.}

1.1 Sample PREPARATION. - We used commercial products without further purification. Toluene was purchased from Merck (Uvasol, spectroscopic grade), n-butanol from SDS (puran), SdS was from Serlabo and sodium chloride from Prolabo.

1.1.1 A-system (salinity scan). - After mixing the constituants in a tube and stirring on a vortex system, the tube was equilibrated in a thermostated bath $\left(T=20 \pm 0.5^{\circ} \mathrm{C}\right)$. The stable demixtion equilibrium was reached after about one day.

Approximate microemulsion compositions were deduced from the measurement of the phase volumes assuming that the phases in excess were pure toluene and/or pure brine (Table I). (The relative precision on the oil and water volume fraction and SdS concentration is about $5 \%$.)
Table I. - Composition of system A, S : salinity $(\%$ by weight of salt in brine); $\phi_{0}$ : toluene volume fraction; $c_{\mathrm{S}}$ : surfactant concentration $\left(\AA^{-3}\right)$ multiplied by $10^{4}$.

\begin{tabular}{|c|c|c|}
\hline$s$ & $\phi_{\circ}$ & $c_{S}$ \\
\hline 3 & .11 & .77 \\
4 & .15 & .74 \\
5 & .19 & .70 \\
6 & .32 & .99 \\
6.5 & .49 & 1.07 \\
7 & .6 & 1.22 \\
7.5 & .71 & 1.11 \\
8 & .81 & .61 \\
9. & .88 & .65 \\
\hline
\end{tabular}

1.1.2 B-system. - The samples were made with a constant salinity brine prepared in advance ( 3 series were tested : $S=5.5 \% ; S=6.5 \% ; S=7.5 \%$ ).

The predetermined volumes of brine $\left(V_{\mathrm{w}}\right)$, toluene, $V_{0}\left(V_{\mathrm{w}}+V_{0}=10 \mathrm{ml}\right)$ and the mass of $\mathrm{SdS}, m_{\mathrm{s}}$ were mixed. Then the samples were «titrated " by repeated addition of small amounts of butanol and stirred. We stopped when the monophasic microemulsion was obtained at equilibrium in a thermostated bath $\left(T=23 \pm 0.5^{\circ} \mathrm{C}\right)$; or, in the opposite case, when there was more than $1 \mathrm{ml}$ of butanol in the tube.

Before complete titration, either we could not solubilize all the SdS, or we obtained a Winsor II microemulsion coexisting with an excess of toluene. As in system A, the B-samples of the inversion zone and of the water rich side are very sensitive to temperature change : a shift of one degree may lead to a demixtion.

Compositions are given in tables II and figure 1.

When the titration was performed, the volume of butanol was less than $8 \%$ of the oil and brine volume. Ignoring the butanol partition coefficient between toluene, brine and film, we neglect butanol volume in the evaluation of the aqueous and oleus volume fractions of the microemulsion $\left(\phi_{\mathrm{w}}\right.$ and $\left.\phi_{0}\right)$. We express $\phi_{\mathrm{w}}$ as $\frac{V_{\mathrm{w}}}{V_{0}+V_{\mathrm{w}}}, \phi_{0}$ as $\frac{V_{0}}{V_{0}+V_{\mathrm{w}}}$ and the surfactant concentration $c_{\mathrm{S}}$ (in $\AA^{-3}$ ) as the ratio of the SdS molecule number to $V_{0}+V_{w}$.

1.1.3C-system. - The preparation procedure was the same as for the system B. The volumes of brine (salinity $S=6.5 \%$ ) and toluene (Table IIc) were 
Table II. - Composition of system B and C, $\phi_{0}:$ toluene volume fraction defined as $V_{0} /\left(V_{0}+V_{\mathrm{w}}\right)(\mathrm{ml}) ; V_{\mathrm{B}}$ : volume of butanol in the microemulsion (in $\mathrm{ml}) ; m_{\mathrm{s}}:$ mass of $\mathrm{SdS}$ in the microemulsion; $c_{\mathrm{S}}:$ surfactant concentration $\left(\AA^{-3}\right)$ multiplied by $10^{4}$.

Table IIa. $-S=6.5 \%, m_{\mathrm{s}}=0.5 \mathrm{~g}$ (this corresponds to a volume of surfactant, $V_{\mathrm{s}}=0.431 \mathrm{ml}$ and a number of SdS molecules $\left.N_{\mathrm{s}}=1.046 \times 10^{21}\right) ; c_{\mathrm{s}}=1.046 \times$ $10^{-4} \AA^{-3}$.

\begin{tabular}{|l|l|}
\hline$\phi_{0}$ & $v_{B}$ \\
\hline .2 & .3 \\
.25 & .36 \\
.3 & .39 \\
.35 & .43 \\
.4 & .46 \\
.45 & .49 \\
.5 & .55 \\
.55 & .59 \\
.6 & .61 \\
.65 & .63 \\
.7 & .65 \\
.75 & .67 \\
.8 & .69 \\
.85 & .71 \\
.9 & .81 \\
\hline
\end{tabular}

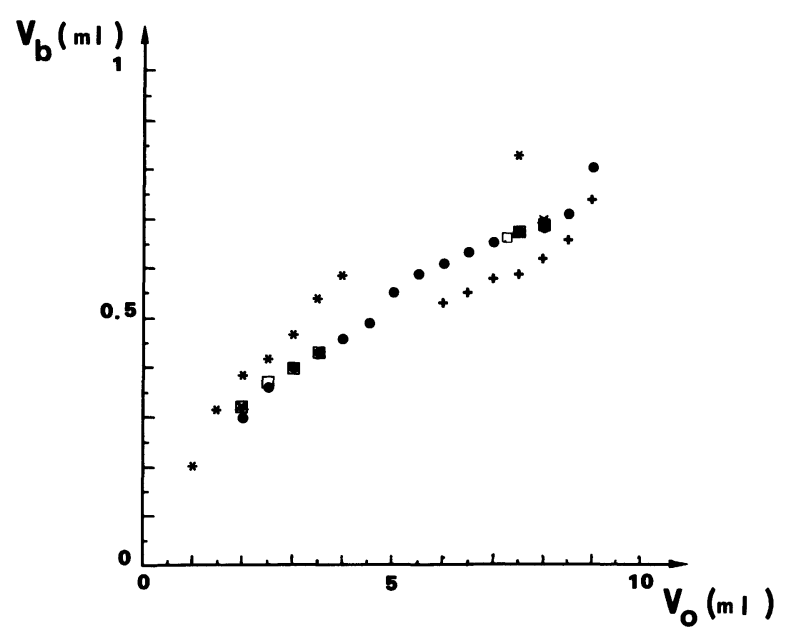

Fig. 1. - Volume of butanol $\left(V_{\mathrm{b}}\right)$ in the B system microemulsions. $\quad S=6.5 \%$; $+S=7.5 \% ; * S=5.5 \%$; $m_{\mathrm{s}}=0.5 \mathrm{~g} ; \square S=6.5 \% ; m_{\mathrm{s}}=0.4 \mathrm{~g} ; \star S=6.5 \%$; $m_{\mathrm{s}}=0.35 \mathrm{~g}$
Table IIb. $-S=6.5 \%, \phi_{0}=0.8$.

\begin{tabular}{|l|l|l|}
\hline \multicolumn{1}{|c|}{$\mathrm{m}_{\mathrm{s}}$} & $\mathrm{c}_{\mathrm{S}}$ & $\mathrm{v}_{\mathrm{B}}$ \\
\hline .35 & .73 & .67 \\
.4 & .84 & .67 \\
.45 & .94 & .68 \\
.5 & 1.05 & .69 \\
.55 & 1.15 & .70 \\
\hline
\end{tabular}

Table IIc. - System C, $m_{\mathrm{s}}=0.5 \mathrm{~g} ; S=6.5 \%$.

\begin{tabular}{|l|l|l|l|l|}
\hline$\phi_{0}$ & $v_{0}$ & $v_{w}$ & $c_{S}$ & $v_{B}$ \\
\hline 0.2 & 1.96 & 7.84 & 1.07 & 0.38 \\
0.3 & 2.24 & 5.23 & 1.4 & 0.30 \\
0.4 & 2.61 & 3.92 & 1.6 & 0.41 \\
0.45 & 2.85 & 3.49 & 1.65 & 0.44 \\
0.5 & 3.14 & 3.14 & 1.67 & 0.43 \\
0.55 & 3.49 & 2.85 & 1.65 & 0.45 \\
0.6 & 3.92 & 2.61 & 1.6 & 0.46 \\
0.7 & 5.23 & 2.24 & 1.4 & 0.53 \\
0.8 & 7.84 & 1.96 & 1.07 & 0.69 \\
\hline
\end{tabular}

chosen so that, with $m_{\mathrm{s}}=0.5 \mathrm{~g}$ and the above definitions of $\phi_{0}, \phi_{\mathrm{w}}$ and $c_{\mathbf{s}}$, one has :

$\xi_{k}=\frac{6 \phi_{0} \phi_{\mathrm{w}}}{c_{\mathrm{S}} \Sigma}=150 \AA$ (with $\Sigma=60 \AA^{2}$, cf. $\S 2.2 .1$ ).

The C-samples $\phi_{0}>0.4$ were prepared at $T=23^{\circ} \mathrm{C}$. They were insensitive to temperature change between $23^{\circ} \mathrm{C}$ and $30^{\circ} \mathrm{C}$. Their X-ray spectra were recorded at $T=23^{\circ} \mathrm{C}$.

After a few days, the two C-samples $\phi_{0}=0.2$ and 0.3 appeared to be not stable at $T=23^{\circ} \mathrm{C}$. Their $\mathrm{X}$-ray spectra were recorded at $T=27^{\circ} \mathrm{C}$, where they were stable without ambiguity.

1.2 ElECTRICAL CONDUCTIVITY MEASUREMENTS. They were performed on system B samples $(S=6.5 \%)$ using a Metrohm E 518 conductimeter. The applied tension frequency varied between $80 \mathrm{~Hz}$ (very low conductivity samples $\sigma<30 \mu \mathrm{S} \mathrm{cm}^{-1}$ ) to $20 \mathrm{kHz}$ (high conductivity samples, $\sigma>10 \mathrm{mS} \mathrm{cm}^{-1}$ ). 
1.3 X-RAY SCATtering EXPERIMENTS. - Two different X-ray sources were used.

The first one was an Elliot rotating copper anode generator (located at the "Centre de Génétique Moléculaire ", Gif-sur-Yvette), giving after monochromation and collimation a very fine beam (thickness $150 \mu \mathrm{m}$ ) about $3 \mathrm{~mm}$ wide. The detector was a position sensitive proportional counter [16]. The X-ray wave length was $\lambda=1.54 \AA$.

The second source was the synchrotron radiations of L.U.R.E. at Orsay. The collimation there was ponctual (beam dimensions $0.5 \mathrm{~mm} \times 1 \mathrm{~mm}$ ). The used wave length was : $\lambda=1.61 \AA$ [17].

All the samples were examined in thin sealed glass capillaries, which diameters had been previously measured. The sample holder was thermostated at the temperature of sample preparation.

Empty capillaries were used to measure the back ground intensity which was subtracted from the sample signal.

In a given series of experiments, the intensities were normalized by dividing the counting rate by the sample thickness, by the sample transmission coefficient and by a quantity proportional to the energy of the incident beam. This defines the experimental intensity $I$.

Experiments which were done with synchrotron radiation will be denoted by $\mathrm{L}$, those with the rotating anode generator by $\mathrm{G}$.

By varying the sample detector distance between $50 \mathrm{~cm}$ and $1 \mathrm{~m}$, we explored the $q$-range $10^{-2}$. $0.25 \AA^{-1}$.

Collimation effects affect the first 50 points (among 200 ) of the $G$ experiment spectra : compared to $L$ spectra the intensities are lowered but the general shape is not changed. As in the very low $q$-range our analysis is only qualitative, the $G$ spectra were not desmeared.

\section{Results and discussion.}

2.1 Remarks ON THE PHASE BeHAVIOUR OF B MICROEMULSIONS. - In system A, oil rich microemulsions are associated with high salinities $(S>6.5 \%)$ and brine rich microemulsions to low salinities. The highest oil and brine uptakes are reached at the inversion point for an "optimal salinity " $S=6.5 \%$. To obtain a toluene volume fraction scan as wide as possible (following the constraint of constant $\mathrm{SdS}$ concentration and salinity), we tried different salinities around the optimal salinity $(S=5.5 \%$, $S=6.5 \%, S=7.5 \%$ ) and different SdS concentrations (see Fig. 1 and Table II).

2.1.1 Salt effect on phase equilibria. - As in system A, the salt effect on the aqueous or oleus character of the microemulsions $\left(m_{\mathrm{s}}=0.5 \mathrm{~g}, V_{0}+V_{\mathrm{w}}=10 \mathrm{ml}\right)$ is striking.

For $S=7.5 \%$ we could only make oil rich microemulsions.
For $S=5.5 \%$, it was the reverse : we made brine rich microemulsions however with much larger amount of butanol, it was also possible to inverse the structure $\left(V_{0}>7.5 \mathrm{ml}\right)$.

Only with the $S=6.5 \%$ brine was it possible to realize microemulsions in the inversion zone and to cover a wide oil volume fraction range $\left(0.2<\phi_{0}<0.9\right)$.

Thus, the optimal salinity not only corresponds to the maximum oil and swater uptakes, but also to the largest existence domain of the microemulsions. 2.1.2 SdS concentration influence on phase equilibria. - At $S=6.5 \%$, we systematically decreased the surfactant amount keeping the oil and brine volumes constant $\left(V_{0}+V_{\mathrm{w}}=10 \mathrm{ml}\right)$. For each toluene volume fraction, a minimum amount of surfactant is always necessary to disperse toluene and brine; below this amount, a demixtion occurs whatever the butanol amount, showing that butanol alone does not replace the surfactant. The smallest is the initial $\phi_{0}$ (or symmetrically $\phi_{w}$ ), the smallest is this lower bound.

2.1.3 Conclusions. - In our systems, the oil and water uptakes (a priori imposed) are mainly determined by SdS and salt.

The Talmon Prager model explains qualitatively why a minimum surfactant amount is necessary. The SdS amount fixes the oil water interfacial area and the size scale of the dispersion; at given oil and water contents, a small specific area corresponds to a large characteristic size (see formula (1)) and a small entropy. If the entropy becomes too small, the surface energy term becomes predominant and the microemulsion demixts.

The salt, in the picture of de Gennes, screens the electrostatic repulsion between SdS polar heads and determines the spontaneous curvature of the interfacial film. It then imposes the dissymmetry of the oil and water contents. At the optimal salinity, the oil and water contents can be varied in the widest proportions showing that the spontaneous curvature of the film is the weakest.

Butanol also influences the uptake, but in a more versatile way. It modulates the film curvature, (very efficiently when at a certain salinity, the film has no preferential curvature towards the oil or water side); however its main rôle is certainly not limited to that. Quite surprisingly, the butanol volume necessary to make a microemulsion of given $\phi_{0}$, $\phi_{\mathrm{w}}$ does not apparently depend on the SdS concentration (Fig. 1 and Table IIb). This could mean that the cosurfactant rôle is not only interfacial in Winsor microemulsions.

2.2 EleCtRICAL CONDUCTIVITY OF B MiCROEMULSIONS. - As a first test of the phase inversion in the toluene volume fraction scan $\left(S=6.5 \%, m_{\mathrm{s}}=0.5 \mathrm{~g}\right.$, $2<V_{0}<9 \mathrm{ml}$ ), we performed electrical conductivity measurements. 
These microemulsions are conducting as soon as $\phi_{\mathrm{w}}$ is greater than $12 \%$. The conductivity $\sigma$ increases as $\phi_{\mathrm{w}}$ increases, in a way very similar to that observed on system A [15] and on other Winsor phases [18] (see Fig. 2, $\sigma$ is normalized by the brine conductivity $\sigma_{\mathrm{w}}=90.7 \mathrm{mS} \mathrm{cm}^{-1}$ ).

In the linear part of the plot, $\frac{\sigma}{\sigma_{\mathrm{w}}}=1.3\left(\phi_{\mathrm{w}}-0.3\right)$. Thus this expression can be compared to an effective medium approximation [19] : $\frac{\sigma}{\sigma_{\mathrm{w}}}=\frac{1}{1-N}(\phi-N)$, where $N$ is the demagnetization coefficient of conducting objects whose volume fraction is $\phi$. This gives $N=0.3 \simeq \frac{1}{3}$ which is the result for a sphere.

We conclude that :

i) The exchange possibilities between brine domains are large.

ii) These domains are not anisotropic.

2.3 Structure of Winsor microemulsions. AnaLYSIS OF THE X-RAY SPECTRA. - When a point collimation of the X-ray beam is used, the experimental intensity $I(q)$ is proportional to $[20,21]$ :

$$
i(q)=4 \pi\left\langle\eta^{2}\right\rangle \int_{0}^{\infty} \gamma(r) r^{2}\left(\frac{\sin q r}{q r}\right) \mathrm{d} r
$$

$i(q)$ is the scattered intensity per unit volume in electronic units. $q$ is the scattering vector related to the scattering angle $2 \theta$ by $q=\frac{4 \pi}{\lambda} \sin \theta$.

If $n(\mathbf{r})$ is the electron density at point $\mathbf{r},\langle n\rangle$ its average value, $\eta(r)=n(r)-\langle n\rangle$ is the local electron-density-fluctuation; its mean-square value is $\left\langle\eta^{2}\right\rangle$ and $\gamma(r)$ is the correlation fonction defined by :

$$
\gamma(r)\left\langle\eta^{2}\right\rangle=\langle\eta(0) \eta(r)\rangle
$$

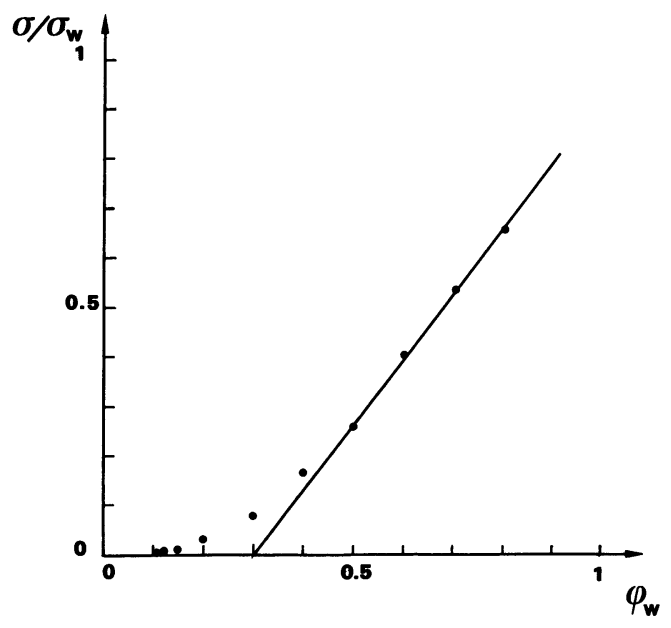

Fig. 2. - Conductivity of microemulsions (system B, $\left.S=6.5 \%, m_{\mathrm{s}}=0.5 \mathrm{~g}\right)$ versus brine volume fraction $\phi_{\mathrm{w}}$. The straight line corresponds to $\sigma / \sigma_{\mathrm{w}}=1.3\left(\phi_{\mathrm{w}}-0.3\right)$. where the brackets mean that the average has been taken.

In our experiments, depending on the series, $q$ varies between $10^{-2}$ and $0.25 \AA^{-1}$ : we explore the microemulsions structure between (roughly) 15 and $300 \AA$.

Because the characteristic sizes $\xi$ of the microemulsions are large $(\xi \geqslant 100 \AA)$, we found that one can observe and define precisely three $q$-ranges :

i) A « large $q$-range » $\left(q>0.1 \AA^{-1}\right.$, small spatial scale, $<30 \AA$ ), also called asymptotic range, since in this domain the X-ray scattering follows general laws (presented below) which are independent of the large scale structure. These laws enable the existence of an interfacial film in our systems to be discussed.

ii) A «medium $q$-range " $(2.5 \leqslant q \xi \leqslant 5)$, where the spectra depend only on the size of the water and/or oil domains.

iii) A very small $q$-range $(q \xi \leqslant 1)$ which describes the large scale structure of the microemulsions.

Only in the last $q$-domain do the spectra exhibit a very qualitative different behaviour from one sample to another.

In the analysis, we will follow this distinction between the three $q$-ranges.

2.3.1 The interfacial film. - At large $q$ (say $\left.0.3>q>0.1 \AA^{-1}\right)$, the signal is due to electron density variations at small spatial scales $(\sim 15-30 \AA)$, smaller than the dimensions of oil and water domains, but larger than the atom sizes. At these scales, if - as it seems - a narrow interfacial film does exist in the microemulsion, one can distinguish three parts : the oil phase and the brine phase, where the electron density is uniform, and the interfacial layer, where the variations of electron density are localized. The intensity then only depends on the local structure of the interface and on the amount of interface.

In particular, if the variations of electron density simply were a jump through the supposed infinitely thin interface from a value $n_{1}$ in brine to $n_{3}$ in toluene, one would have :

$$
\lim _{q \rightarrow \infty} q^{4} i(q)=2 \pi \frac{A}{V}\left(n_{3}-n_{1}\right)^{2} \quad \text { (Porod's law [22]) }
$$

$A / V$ is the area per unit volume between oil and water.

However the intensities of the samples do not obey Porod's law (3), but they exhibit a definite asymptotic behaviour.

i) This behaviour only depends on the surfactant concentration, $c_{\mathrm{S}}$ (we checked in particular that it was identical for all B samples of the toluene scan, at constant $c_{\mathrm{S}}$ ).

ii) $q^{4} I$ is not constant but linear in $q^{2}$ (Fig. 3). We have $q^{4} I \simeq Y+P q^{2} ; Y$ and $P$ are both positive. Within the experimental uncertainty, the slope $P$ of the plot $q^{4} I$ versus $q^{2}$ and the intercept with $y$ 


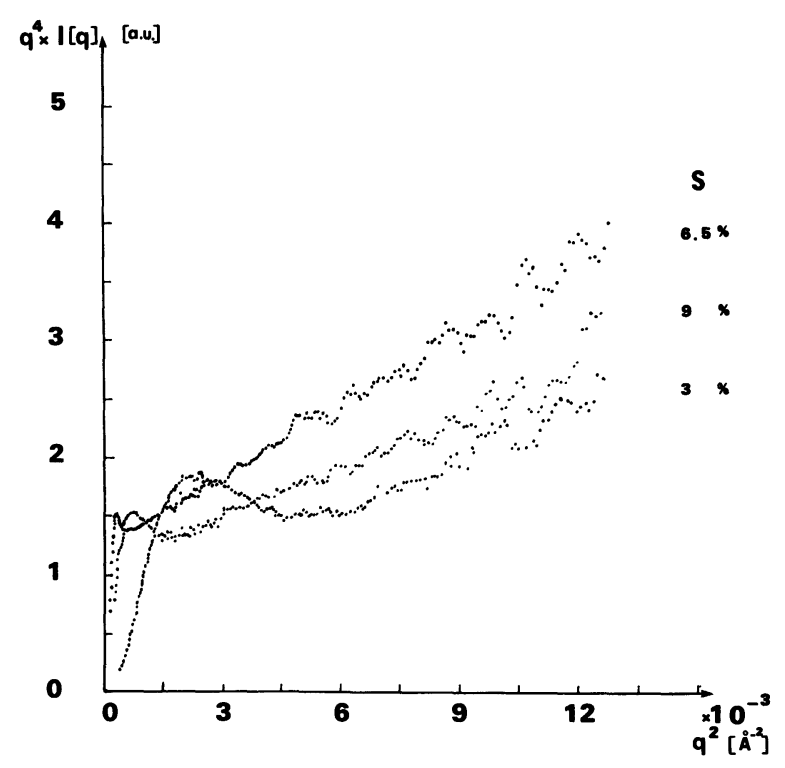

Fig. 3. $-q^{4}$ I versus $q^{2}$. A-system (L experiment). From the 100th point, the data are averaged on five consecutive channels. $S=3 \%, S=6.5 \%, S=9 \%$.

axis, $Y$ are both proportional to the SdS concentration (see Table III).

This behaviour is at variance with that expected with a critical molecular mixture, where the OrnsteinZernike law predicts $i(q) \sim 1 / q^{2}$ without a $1 / q^{4}$ term. In contrast, the existence of a well defined interfacial surfactant film between oil and water enables all the above facts, to be interpreted if one takes into account the polar heads electron density contribution to the contrast.

With the electron density profile of figure 4 , for a locally flat interface, Porod's law takes the form (Appendix I) :

$$
\begin{aligned}
& \lim _{q d<1} q^{4} i(q)=2 \pi \frac{A}{V}\left(n_{3}-n_{1}\right)^{2} \times \\
& \times\left(1+\frac{\left(n_{2}-n_{1}\right)\left(n_{2}-n_{3}\right)}{\left(n_{3}-n_{1}\right)^{2}}(q d)^{2}+0(q d)^{4}\right)
\end{aligned}
$$

where $d$ is the thickness of the polar part of the interfacial film $n_{1}, n_{2}$ and $n_{3}$ respectively are the electron densities of brine, film and toluene.

The first term is Porod's term (3) due to the difference of electron density between oil and water; the second is the contribution of the thin polar heads sheet $\left(i(q) \sim 1 / q^{2}\right.$ is characteristic of the scattering by thin disks [20]). Both terms are surface terms, proportional to the specific area, which is fixed by the surfactant amount. If all the SdS is at the oil/water interface, $A / V=c_{\mathrm{S}} \Sigma(\Sigma$ is the area per SdS polar head), and the two coefficients $P$ and $Y$ of the law $q^{4} I=Y+P q^{2}$ are proportional to $c_{\mathrm{S}} \Sigma$. Up to the experimental uncertainty, this is indeed the case and $\Sigma$ is constant.
Table III. - Parameters of the asymptotic behaviour of $I(q)$ (defined in text). (a) $\mathrm{L}_{1}$ experiment. A-system and two samples of the B-system $\left(S=6.5 \%, m_{\mathrm{s}}=\right.$ $0.5 \mathrm{~g}, \phi_{0}=0.5$ and $\phi_{0}=0.75$ ); (b) $\mathrm{G}_{3}$ experiment.

\begin{tabular}{|c|c|c|c|c|c|}
\hline s & $\mathrm{P}\left(\mathrm{a} . \mathrm{u}_{\text {. }}\right)$ & ${ }^{\mathrm{P}} / 10^{4} \mathrm{c} \mathrm{s}$ & $Y_{0}$ (a.u.) & $\mathrm{Y}_{0} /{ }_{10^{4} \mathrm{cs}}$ & $\ell(\AA)$ \\
\hline 3 & .12 & .16 & $910^{-4}$ & $1.210^{-3}$ & 11.6 \\
\hline 4 & .12 & .16 & $9,10^{-4}$ & $1.210^{-3}$ & 11.6 \\
\hline 5 & .12 & .17 & $910^{-4}$ & $1.310^{-3}$ & 11.6 \\
\hline 6 & .20 & .20 & $1.310^{-3}$ & $1.310^{-3}$ & 12.4 \\
\hline 6.5 & .20 & .19 & $1.310^{-3}$ & $1.210^{-3}$ & 12.4 \\
\hline 7 & .20 & .16 & $1.310^{-3}$ & $1.110^{-3}$ & 12.4 \\
\hline 7.5 & .20 & .18 & $1.310^{-3}$ & $1.210^{-3}$ & 12.4 \\
\hline 8 & .14 & .23 & $10^{-3}$ & $1.610^{-3}$ & 11.8 \\
\hline 9 & .14 & .22 & $10^{-3}$ & $1.510^{-3}$ & 11.8 \\
\hline$\phi_{0}$ & & & & & \\
\hline 0.5 & .22 & .21 & $1.410^{-3}$ & $1.310^{-3}$ & 12.5 \\
\hline 0.75 & .22 & .21 & $1.410^{-3}$ & $1.3 \quad 10^{-3}$ & 12.5 \\
\hline
\end{tabular}
System C.

b

\begin{tabular}{|l|l|l|l|l|l|}
\hline$\phi_{0}$ & $\mathrm{P}$ & $\mathrm{P} / 10^{4} \mathrm{c} \mathrm{S}$ & $\mathrm{Y}$ & $\mathrm{Y} / 10^{4} \mathrm{c} \mathrm{S}$ & $\mathrm{l}$ \\
\hline .2 & .14 & .13 & $910^{-4}$ & $0.810^{-3}$ & 12.6 \\
.3 & .17 & .12 & $1.310^{-3}$ & $0.910^{-3}$ & 11.4 \\
.4 & .19 & .12 & $1.710^{-3}$ & $1.110^{-3}$ & 10.6 \\
.45 & .22 & .13 & $1.410^{-3}$ & $0.910^{-3}$ & 12.4 \\
.5 & .25 & .15 & $1.710^{-3}$ & $1.010^{-3}$ & 12 \\
.55 & .24 & .14 & $1.710^{-3}$ & $1.010^{-3}$ & 11.8 \\
.6 & .23 & .14 & $1.410^{-3}$ & $0.910^{-3}$ & 12.7 \\
.7 & .22 & .16 & $1.410^{-3}$ & $1.010^{-3}$ & 12.5 \\
.8 & .17 & .16 & $910^{-4}$ & $0.810^{-3}$ & 13.6 \\
\hline
\end{tabular}

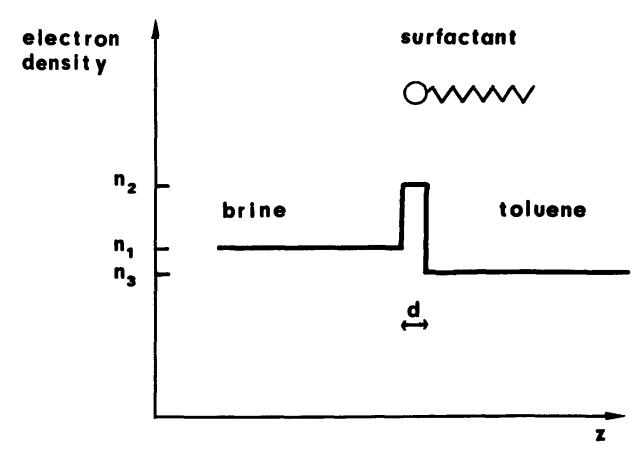

Fig. 4. - Electron density profile through the interfacial layer.

In formula (4), the ratio $P / Y$ is :

$\frac{P}{Y}=\frac{\left(n_{2}-n_{1}\right)\left(n_{2}-n_{3}\right)}{\left(n_{3}-n_{1}\right)^{2}} d^{2}=l^{2} \quad(l$ is a length $)$. 
The measured value of $l$ does not vary from a sample to another

$$
l=12 \pm 1.5 \AA .
$$

If one knows $d$, one deduces from $l$ the electron excess in the polar part of the interfacial film and the area per SdS polar head $\Sigma$ (in $\AA^{2}$ ) (Appendix II).

As a reference, we chose a brine in cyclohexanepentanol microemulsion, whose structure has been already studied by neutron scattering (6) (it is called $5 \mathrm{~B} 1 \mathrm{M}$ in this reference : the $\mathrm{NaCl}$ brine molarity is $1 \mathrm{M}(S=5.5 \%)$, the weight ratio of water to $\mathrm{SdS}$ is 2.5 , the cyclohexane-brine volume ratio is 5). This microemulsion is of the hard sphere type [6]. We measured its X-ray spectrum and observed that $q^{4} I$ was also linear in $q^{2}$ in the asymptotic range as in our brine-toluene microemulsions.

Certainly $d$, the thickness of the SdS polar heads layer is not very different in the two systems. Knowing $\Sigma$ for the B1M system from the neutron scattering experiments, we deduce the value of $d$ in this system from the X-ray experiments, and use the obtained value to evaluate $\Sigma$ in our brine-toluene system.

The expression of $l$ calculated from the electron density of brine and cyclohexane is (Appendix II)

$$
l^{2}=\left(\frac{470}{\Sigma}\right)^{2}\left(1+\frac{\mathrm{d} \Sigma}{470}\right) \text {. }
$$

Experimentally, we found $l=11 \AA$. The $\Sigma$ value deduced from the water droplets radius measurements is $52 \AA^{2}$ [6]. This is compatible with a thickness $d=5 \AA$, comparable to both the Debye screening length and polar heads size.

Returning to the brine-toluene microemulsions, with $d=5 \AA, l=12 \pm 1.5 \AA, l^{2}=\left(\frac{595}{\Sigma}\right)^{2}\left(1+\frac{\Sigma d}{595}\right)($ at salinity $S=6.5 \%$ ), we deduce, $\Sigma=61 \pm 8 \AA^{2}$, in accordance with the known results [15].

Thus, the oil-water interface in our systems is narrow and well defined, even in the critical behaviour zone of the phase diagram.

Our study proves that the determination of the asymptotic behaviour of the intensity scattered by microemulsions is very useful to discriminate between the two possible situations : microemulsion, molecular solubilized mixture or microemulsion, dispersed composite liquid. Apparently, this mean was not used before, because the X-ray or neutron scattering measurements (which can offer simpler two-phases contrasts) were not extended to sufficiently large $q$.

To our knowledge, such a type of asymptotic behaviour $-q^{4} I$ linear in $q^{2}-$ is observed here for the first time in colloidal surfactant systems. In the $\mathrm{X}$-ray experiments, this is due to the high contrast of SdS polar heads (relatively to water and toluene) associated with large sizes (much larger than the thickness of the interfacial film). This enables the asymptotic regime to be observed at relatively small values of $q\left(q>0.1 \AA^{-1}\right)$.
Such a type of contrast allows a practical determination of $\Sigma$ which is :

i) independent of the structure model of microemulsions,

ii) independent of the normalization of the intensities. (Notice that one cannot normalize $I(q)$ with the usual invariant $\int_{0}^{\infty} q^{2} I(q) \mathrm{d} q[21]$, which in this case diverges).

2.3.2 Semi-local scale : characteristic length. Expressions (3) and (4) of Porod's law are valid as long as the interface is flat on the scale defined by the scattering angle $\left(\sim \pi q^{-1}\right)$. In the absence of a detailed structural model adapted to our contrast conditions, our idea is that the location of the $q$ crossover zone between the very small angle behaviour and the asymptotic behaviour gives the size scale where the film becomes curved, i.e. an estimation of the persistence length of the film, $\xi_{k}$.

In the paragraph (a) below, we define the cross-over scattering vector $q_{1}$ and point out the practical and theoretical limitations of this definition. In paragraph (b), we show that, for all the samples, the intensities in the medium $q$-range can be written as $I(q)=I_{0} f\left(q / q_{1}\right)$, thus $q_{1}$ is a good choice to determine a characteristic length. In paragraph (c), we report the observed dependence of $q_{1}$ on the composition of the microemulsions.

a) The definition of a characteristic scattering vector $q_{1}$. We have chosen as a definition of the cross-over scattering vector $q_{1}$, the abscissa of the minimum of the $q^{4} I$ versus $q$ plot (see Fig. 5 and Table IVa, b, c, d).

Let us first point out the practical limitations of this choice. The determination of $q_{1}$ mainly depends on the precision on $q^{4} I\left(\frac{\delta q}{q} \sim 2 \%\right.$ and $\frac{\delta I}{I} \sim 2 \%$ lead to $\left.\frac{\delta q^{4} I}{q^{4} I} \sim 10 \%\right)$. We found that the measure-

Table IV. - Structural data of A, B, C samples, $q_{1}$ is defined in text, $\Sigma=6 \times 10^{1} \AA^{2}, \phi$ is $\phi_{\mathrm{w}}$ in the oil rich side and $\phi_{0}$ in the water rich side.

Table IVa. - System A.

\begin{tabular}{|l|l|l|l|l|}
\hline$s$ & $q_{1}\left(\AA^{-1}\right)$ & $q_{1}^{-1}(\AA)$ & $c s^{\sum q_{1}^{-1}}$ & $c s^{\sum q_{1}^{-1} \phi^{-1}}$ \\
\hline 3 & .073 & 13.7 & .06 & .55 \\
4 & .058 & 17.3 & .0 .75 & .5 \\
5 & .041 & 24.6 & .10 & .53 \\
6 & .03 & 33.7 & .20 & .63 \\
6.5 & .024 & 41.1 & .26 & .53 \\
7 & .025 & 40 & .29 & .73 \\
7.5 & .029 & 35.1 & .23 & .79 \\
8 & .033 & 30.8 & .11 & .58 \\
9 & .041 & 24.5 & .095 & .79 \\
\hline
\end{tabular}




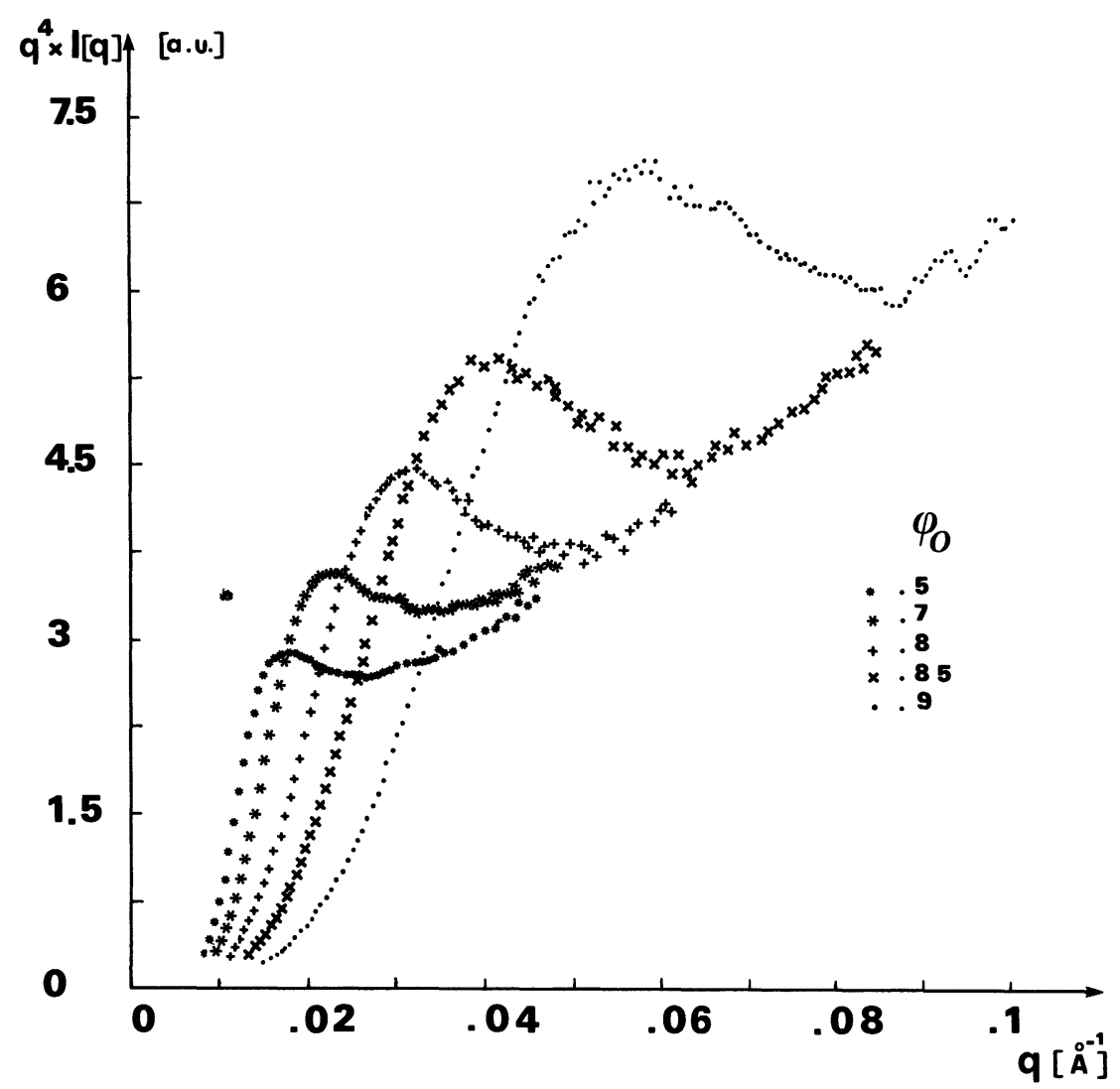

Fig. 5. $-q^{4}$ I versus $q$. System $\mathrm{B}\left(\mathrm{L}_{2}\right.$ experiment $) ; S=6.5 \%, m_{\mathrm{s}}=0.5 \mathrm{~g} ; * * \phi_{0}=0.5, * * 0.7,++0.8, \times \times 0.85, \bullet \bullet 0.9$.

Table IVb. - System B, oil volume fraction scan $\left(S=6.5 \%, m_{\mathrm{s}}=0.5 \mathrm{~g}\right)$, first column : G experiment; second column : L experiment.

\begin{tabular}{|c|c|c|c|c|c|c|c|c|}
\hline$\phi_{0}$ & $q_{1}$ & & $q_{1}^{-1}$ & & $c s^{\sum q_{1}^{-1}}$ & & $c_{s} \sum q_{1}^{-1} \phi^{-1}$ & \\
\hline .2 & .042 & & 23.8 & & 15 & & .75 & \\
\hline .25 & .035 & & 28.6 & & .18 & & .72 & \\
\hline .3 & .036 & & 27.8 & & .17 & & .57 & \\
\hline .35 & .032 & & 31.3 & & .195 & & .56 & \\
\hline .4 & .029 & & 34.5 & & .215 & & .54 & \\
\hline .45 & .028 & & 35.7 & & .225 & & .5 & \\
\hline & .025 & .026 & $40^{\circ}$ & 37.8 & .25 & .24 & .5 & .48 \\
\hline .55 & .027 & .027 & 37 & 37.1 & .23 & .23 & .51 & .51 \\
\hline .65 & .036 & .029 & 27.8 & 34.3 & .175 & .215 & .5 & .61 \\
\hline .7 & .04 & .035 & 25 & & .155 & .18 & .52 & .6 \\
\hline .75 & .043 & .039 & 23.3 & 25.6 & .145 & .16 & .58 & .64 \\
\hline .8 & $\begin{array}{l}.052 \\
.052\end{array}$ & .050 & 19.2 & $20^{\circ}$ & .12 & .125 & .6 & .63 \\
\hline .85 & .065 & .063 & $\begin{array}{l}15.4 \\
\end{array}$ & 16 & .095 & .10 & .63 & .67 \\
\hline 9 & .085 & .087 & 11.8 & 11.5 & .075 & .07 & .75 & .07 \\
\hline
\end{tabular}

Table IVc. - System B, SdS concentration scan $(S=$ $\left.6.5 \%, \phi_{0}=0.8\right)(\mathrm{L}$ experiment $)$.

\begin{tabular}{|c|c|c|c|c|}
\hline $\mathrm{m}_{\mathrm{s}}$ & $\mathrm{q}_{1}$ & $\mathrm{q}_{1}^{-1}$ & $\mathrm{c}_{\mathrm{S}} \Sigma \mathrm{q}_{1}^{-1}$ & $\mathrm{c}_{\mathrm{S}} \Sigma \mathrm{q}_{1}^{-1} \phi_{\mathrm{w}}$ \\
\hline .35 & .033 & 30.8 & .135 & .68 \\
.4 & .039 & 25.8 & .13 & .65 \\
.45 & .044 & 22.7 & .13 & .65 \\
.5 & .05 & 20 & .125 & .63 \\
.55 & .055 & 18.2 & .125 & .63 \\
\hline
\end{tabular}

Table IVd. - System C. « constant $\xi » \operatorname{scan}(S=6.5 \%)$. $\frac{6 \phi_{0} \phi_{\mathrm{w}}}{c_{\mathrm{s}} \Sigma}=150 \AA$.

\begin{tabular}{|l|l|l|l|}
\hline$\phi_{0}$ & $\mathrm{q}_{1}$ & $\mathrm{q}_{1}{ }^{-1}$ & $\mathrm{c}_{\mathrm{s}^{\Sigma \mathrm{q}_{1}}}{ }^{-1}$ \\
\hline .2 & .051 & 19.6 & .125 \\
.3 & .04 & 25 & .21 \\
.4 & .046 & 21.7 & .21 \\
.45 & .046 & 21.7 & .215 \\
.5 & .047 & 21.3 & .215 \\
.55 & .046 & 21.7 & .215 \\
.6 & .046 & 21.7 & .21 \\
.7 & .050 & 20 & .17 \\
.8 & .052 & 19.2 & .12 \\
\hline
\end{tabular}

ment of $q_{1}$ was more precise in $\mathrm{L}$ experiments than in $\mathrm{G}$ experiments : the statistics are better and the fluctuations of the detector response are smaller during the (about 10 times) smaller counting time. Globally, considering that the larger relative uncertainties on the low $q$ values are compensated by the 
smaller statistical uncertainties on the intensities, we estimate the precision $\delta q_{1} / q_{1}$ to be about $5 \%$ in $\mathrm{L}$ experiments and $10 \%$ in $\mathrm{G}$ experiments. By comparing the results obtained on the two set-up (Table IV and Fig. 6), we find that the systematic error introduced in the $G$ experiments by the collimations corrections are within the above uncertainties.

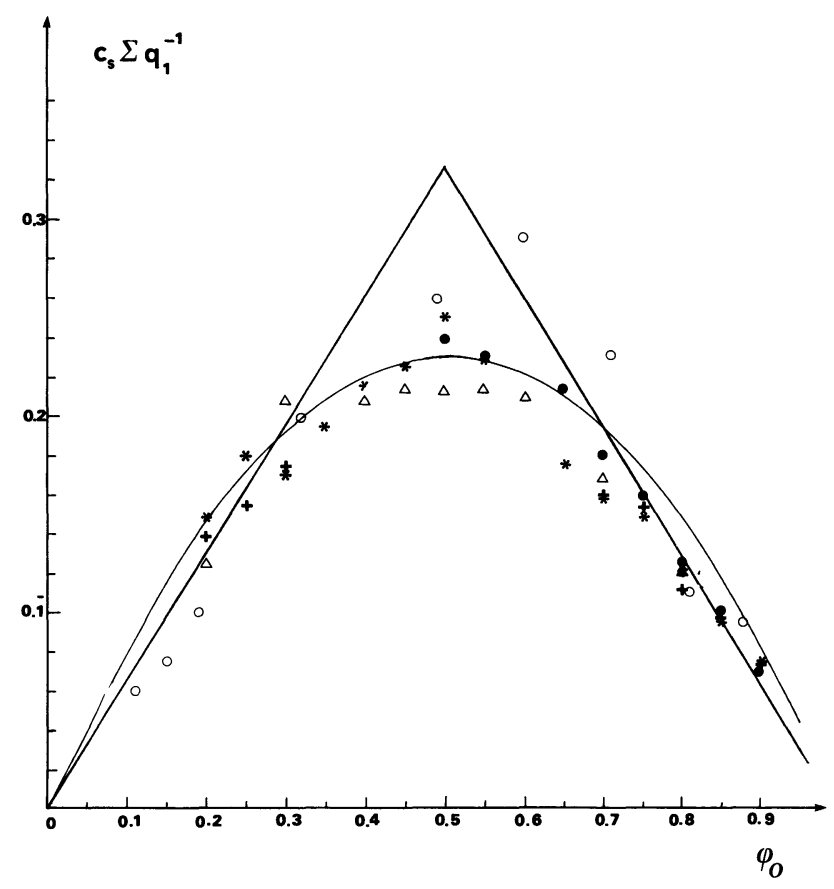

Fig. 6. - The product $c_{\mathrm{S}} \Sigma q_{1}^{-1}$ is plotted versus the toluene volume fraction $\phi_{0}$. O system $A\left(L_{1}\right.$ experiment); o system $B$ $\left(\mathrm{L}_{2}\right.$ experiment $) ;+$ system $\mathrm{B}\left(\mathrm{G}_{1}\right.$ experiment $) ; *$ system $\mathrm{B}$ $\left(G_{2}\right.$ experiment $) ; \Delta$ system $C\left(G_{3}\right.$ experiment $)$. The straight line corresponds to $c_{\mathrm{S}} \Sigma q_{1}^{-1}=0.65\left(\phi_{\mathrm{w}}\right.$ or $\left.\phi_{0}\right)$. The parabola corresponds to $c_{\mathrm{S}} \Sigma q_{1}^{-1}=0.9 \phi_{0} \phi_{\mathrm{w}}$.

There is also a theoretical limitation in our choice : $q_{1}$ is located in a $q$-range where the volume-scattering contribution (dominated at low $q$ by oil and water correlations) a priori becomes of the same order of magnitude as the surface contribution (dominated at high $q$ by the film). A priori $q_{1}$ may be a complicated function of the characteristic size $\xi$, the oil, water, SdS volume fractions and electron densities. However there are very interesting particular cases where the relation between $q_{1}$ and $\xi$ is simple. If there is only one length in the structure description and if one can write the intensities as $i(q)=i_{0} f(q \xi)$, then $q_{1}^{-1}$ is proportional to $\xi$. This occurs :

i) If the film contribution is negligible. Then Porod's law (3) imposes

$$
i(q) \sim \frac{\left(n_{1}-n_{3}\right)^{2}}{q^{4}} c_{\mathrm{S}} \Sigma \sim \frac{i_{0}}{q^{4} \xi^{4}}
$$

and

$$
i_{0} \sim\left(n_{1}-n_{3}\right)^{2} c_{\mathrm{S}} \Sigma \xi^{4}
$$

ii) If the film contribution is dominant. Then, Porod's law (4) imposes

$$
\begin{aligned}
i(q) & \sim \frac{\left(n_{2}-n_{1}\right)^{2}}{q^{2}} c_{\mathrm{S}} \Sigma d^{2} \sim \frac{i_{0}}{q^{2} \xi^{2}} \\
i_{0} & \sim\left(n_{2}-n_{1}\right)^{2} c_{\mathrm{S}} \Sigma d^{2} \xi^{2} .
\end{aligned}
$$

b) Behaviour of the intensities in the medium $q$-range $\left(q \sim q_{1}\right)$.

i) Similarity of the spectra. - As the relation between $q_{1}$ and the characteristic length could be not simple, we looked for the existence of a scaling law by plotting $\operatorname{Ln} I$ as a function of $\left(q / q_{1}\right)^{2}$. For $0.5<q / q_{1}<1$ (domain of the $q^{4} I$ versus $q$ peak), the logarithms of the intensities only differ by a constant (Fig. 7 concerning system B), the spectra are similar. The similarity domain is even more extended towards smaller $q$ for the dilutest samples $\left(\phi_{0} \geqslant 0.8\right)$. Thus experimentally $q_{1}$ is a good scattering vector unit in the medium $q$-range. For $0.5<$ $q / q_{1}<1$, we write $I(q)=I_{0} f\left(q / q_{1}\right)$.

ii) Variations of the intensities. - The formulae (5) and (6) predict the variations of $I_{0}$, prefactor of the scaling law, as a function of the surfactant concentration $c_{\mathrm{s}}$ and the characteristic length $\xi$, in the two extreme cases : the film contribution either dominant or negligible. Assuming $q_{1}^{-1} \sim \xi$, we found that the measured $I_{0}$ decreases with increasing $q_{1}$, but in a way which is not so simple as that stated by formulae (5) and (6).

The mean slope of a plot $\ln I_{0}$ versus $\ln q_{1}$ is about - 3.3 for the scan $\phi_{0}$ variable at constant surfactant concentration, i.e. intermediate between the prediction -4 (film contribution negligible) and -2 (film contribution dominant).

At $c_{\mathrm{s}}$ variable (serie $\phi_{0}=0.8$ ), the same slope is about -2.6 ; with the assumption that $\xi$ is inversely proportional to $c_{\mathrm{S}}$, the predictions of formulae (5) and (6) are in this case, respectively, $I_{0} \sim(1 / \xi)^{-3}$ or $(1 / \xi)^{-1}$.

Thus, we are not strictly in one of the two pure cases of formulae (5) and (6), however the evolution of $I_{0}$ is better described by formula (5) than by formula (6). We conclude that the oil-water contribution to the scattering is the most important at $q$ less than $q_{1}$, and that, in a first approximation, $q_{1}^{-1}$ fulfills the conditions to be the characteristic size of our microemulsions.

iii) Remark. - A remarkable feature of the plots ln $I$ versus $\left(q / q_{1}\right)^{2}$ is that, the curves are approximately linear in the range $0.2<\left(q / q_{1}\right)^{2} \leqslant 0.65$ (Fig. 7), although it is not the very low $q$ Guinier range [20] (the Guinier law $I(q) \sim \exp \left(-\frac{q^{2} R_{\mathrm{G}}^{2}}{3}\right)\left(q R_{\mathrm{G}}<1\right)$ is encountered in very dilute solutions of non-interacting 


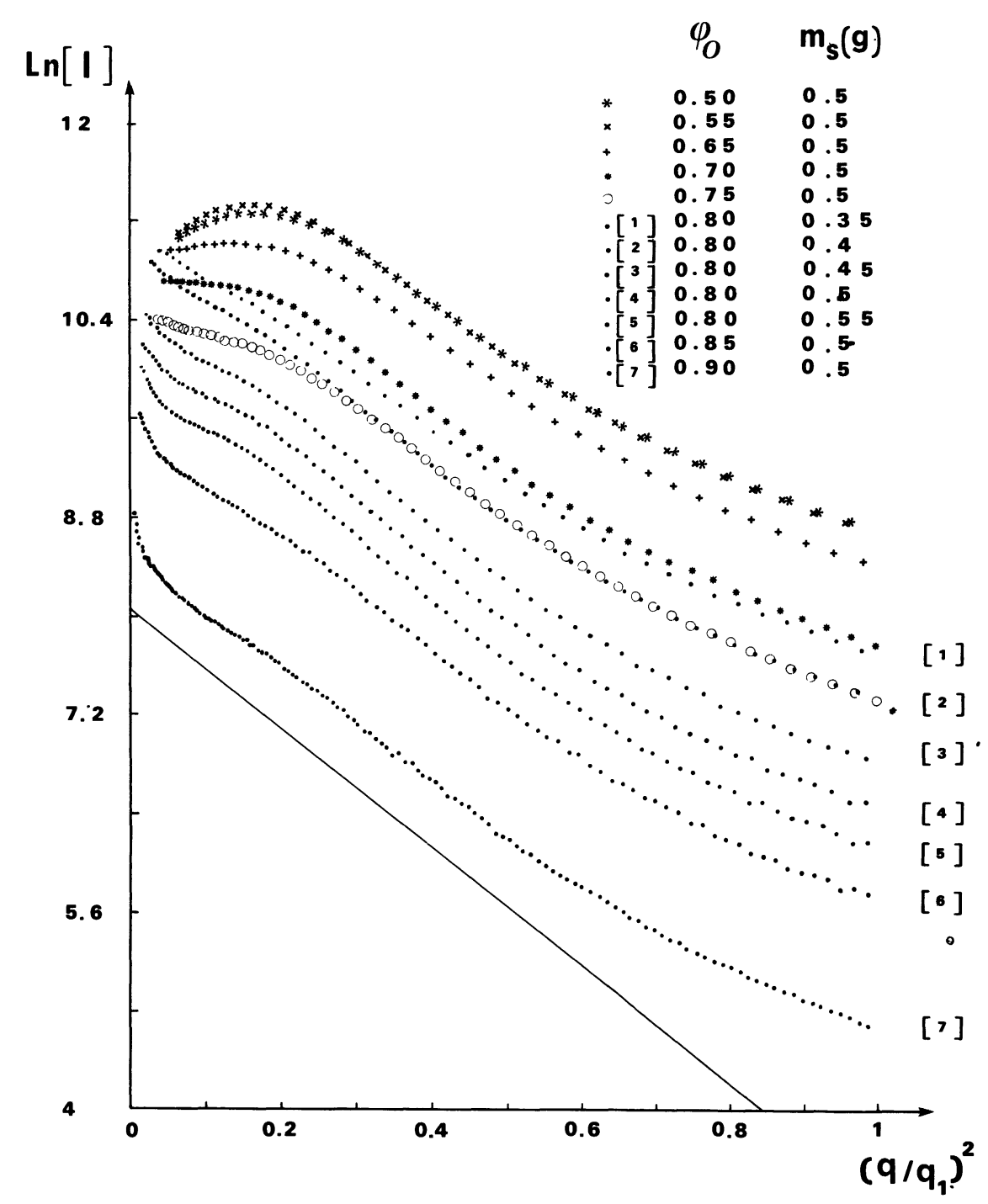

Fig. 7. - Intensity in reduced unit $\left(q / q_{1}\right)\left(\ln I v s .\left(q / q_{1}\right)^{2}\right)$ system B $\left(\mathrm{L}_{2}\right.$ experiment).

particles, which have a radius of gyration $R_{\mathrm{G}}$ ). A possible explanation of this fact is that the expression $q^{4} \exp \left(-\frac{q^{2} L^{2}}{3}\right)$ well reproduces the peak of the $q^{4} I$ versus $q$ plot. This can serve as another means to estimate the typical size in the systems. On figure 7, the straight line parallel to the experimental curves corresponds to the expression $I \sim \exp \left(-\frac{q^{2} L^{2}}{3}\right)$ with $L=3.8 q_{1}^{-1}$.

c) Behaviour of $q_{1}$ : interpretation. - At constant salinity $(S=6.5 \%)$ and oil volume fraction $\left(\phi_{0}=0.8\right)$, we have varied the SdS concentration. In this series $q_{1}$ varies linearly as a function of $\mathrm{SdS}$ concentration $c_{\mathrm{S}}$ (Table IVc).

In system $\mathrm{C}$, where the theoretical length $\xi_{k}=\frac{6 \phi_{0} \phi_{\mathrm{w}}}{c_{\mathrm{S}} \Sigma}$ was purposely held constant $\left(\xi_{k}=150 \AA\right.$ with $\left.\Sigma=60 \AA^{2}\right) . q_{1}$ is found to be constant ( $q_{1}=$ $\left.0.046 \AA^{-1}\right)$ in the inversion zone $\left(0.4 \leqslant \phi_{0} \leqslant 0.6\right)$.

To study the behaviour of $q_{1}$ as a function of $\phi_{0}$, we have plotted for all samples of system A (salinity scan) and systems B and C ( $\phi_{0}$-scans), the product $c_{\mathrm{S}} \Sigma q_{1}^{-1}$ versus $\phi_{0}$ (Fig. 6). The resulting curve is symmetric in $\phi_{0}$ and maximum at the inversion point $\left(\phi_{0}=0.5\right)$. On the water rich side $\left(\phi_{0}<0.3\right), c_{\mathrm{s}} \Sigma q_{1}^{-1}$ is linear in $\phi_{0}$. On the oil rich side $\left(\phi_{0}>0.7\right), c_{\mathrm{S}} \Sigma q_{1}^{-1}$ is linear in $\left(1-\phi_{0}\right)$.

Our interpretation is the following :

- At low water or oil concentrations $\left(\phi_{0}\right.$ or $\phi_{\mathrm{w}}<0.3$ ), $q_{1}^{-1}$ is proportional to the size of distinct isotropic objects.

Their size $R$ is fixed by their volume to surface ratio and one has

$$
q_{1}^{-1} \sim R \sim \frac{\phi_{0}}{c_{\mathrm{S}} \Sigma} \quad\left(\text { or } \phi_{\mathrm{w}}\right) .
$$


Using the low water concentration data $\left(\phi_{\mathrm{w}}<0.3\right)$ (Table IVb and c) the proportionality factor between $q_{1}^{-1}$ and $\frac{\phi_{0}}{c_{\mathrm{S}} \Sigma}$ is experimentally :

$$
\frac{c_{\mathrm{S}} \Sigma}{\phi_{\mathrm{w}}} q_{1}^{-1}=0.65 \pm 0.03
$$

(we neglect the slight dissymmetry between the oil rich and water rich side).

In a sphere model $R_{\mathrm{S}, \mathrm{w}}=\frac{3 \phi_{\mathrm{w}}}{c_{\mathrm{S}} \Sigma}$ thus the phenomenological relation between $R_{\mathrm{S}, \mathrm{w}}$ and $q_{1}$ is $R_{\mathrm{S}, \mathrm{w}}=4.6 q_{1}^{-1}$. Surprisingly the experimental relation between $L$ (defined in the precedent paragraph) and $R_{\mathrm{S}}$ : $L=3.8 q_{1}^{-1}=0.8 R_{\mathrm{S}}$, is akin to the expression of the radius of gyration $R_{\mathrm{G}}$ of a sphere of radius $R: R_{\mathrm{G}}=$ $0.77 R$.

We also have checked the procedure of size measurement by $q_{1}$ on the known microemulsion 5B $1 \mathrm{M}$ of reference [6]. We found $q_{1}=0.063 \AA^{-1}$ and $4.6 q_{1}^{-1}=$ $73 \AA$. The radius of the water core $r_{\mathrm{w}}$ deduced from neutron scattering is $66.5 \AA$. Again the agreement is good.

As our results are consistent at low oil or water concentration, we infer that a proportionality relation between $q_{1}^{-1}$ and the characteristic length of the microemulsions is also valid in the inversion zone.

- In the inversion zone $\left(0.3<\phi_{0}<0.7\right)$, as directly demonstrated by the results on system $\mathrm{C}$ (constant $\xi_{k}$ scan) which confirm the evolution on system B ( $\phi_{0}$ scan), $q_{1}^{-1}$ follows - up to a numerical factor - the prediction (1) (we do not know if the deviations observed on system A (salinity scan) - figure 6are significant because of the much larger uncertainty on the phase composition). Experimentally, one approximately has $q_{1}^{-1}=0.9 \frac{\phi_{0} \phi_{\mathrm{w}}}{c_{\mathrm{S}} \Sigma}$. In the inversion zone, the experimental relation between $q_{1}^{-1}$ and $\xi_{k}$ (defined by (1)) is then $q_{1}^{-1}=0.15 \xi_{k}$ or $\xi_{k}=6.5 q_{1}^{-1}$. Then $L=3.8 q_{1}^{-1}=0.6 \xi_{k}$.

Thus, although not a direct proof, the observed relation between $q_{1}$ and $\xi_{k}$, showing that in our systems the inversion is a smooth process culminating at $\phi_{0}=0.5$, may be the first experimental evidence from scattering experiments that as $\phi_{0}$ increases, one passes progressively from a structure made of distinct oil in water spheres to the bicontinuous structure described by the Talmon-Prager or de Gennes model; the curvature of the film is inverted at $\phi_{0}=0.5$, and, as $\phi_{0}$ again increases, the reverse process takes place.

2.3.3 CORRELATIONS OR INTERACTIONS IN MICROEMULsions. - The structures of our microemulsion are very similar (up to a scale factor) at scales which are of the same order of magnitude or smaller than the characteristic size. The differences between the samples appear at larger scales (smaller scattering vector $q \sim 10^{-2} \AA^{-1}$ ). We have noticed three different types of behaviour : i) The B samples in the inversion zone $\left(0.4 \leqslant \phi_{0} \leqslant\right.$ $0.65)$ exhibits a diffuse band centred around $q^{*}$ $\left(q^{*}=0.011 \AA^{-1}\right.$, the corresponding Bragg spacing $d^{*}=2 \pi q^{*-1}$ is $590 \AA$ ) [23]. This peak is broadened, but does not move as one goes far away from the inversion point and it disappears at $\phi_{0}=0.7$ (oil rich side) and $\phi_{0}=0.35$ (water rich side). The same features were observed in the $\mathrm{C}$ system $\left(q^{*}=0.02 \AA^{-1}\right.$, $\left.d^{*}=310 \AA\right)$ revealing a strong correlation between the place of the peak and $q_{1}$.

ii) The $B$ samples outside the inversion zone $\left(\phi_{0} \leqslant 0.3, \phi_{0} \geqslant 0.75\right)$ do not follow the "Guinier " law [20] in the Guinier range $q R \leqslant 1$ (Fig. 7). In particular, the intensities rapidly increase as $q$ goes to zero.

iii) The two samples most dilute in oil of system $\mathrm{A}$ ( $S=3 \%$ and $S=4 \%$ ) also exhibit a diffuse peak. $\left(q^{*}(S=3 \%)=0.25 \AA^{-1}, \quad d^{*}(3 \%)=250 \AA\right.$, $\left.q^{*}(S=4 \%)=0.019 \AA^{-1}, d^{*}(4 \%)=330 \AA\right)$.

These three different behaviours indicate that the interactions or correlations between oil or water domains are quite complex.

i) In the inversion zone, the intensity scattered by a two phase random oil and water partition in Voronoi polyhedra has been calculated by Kaler and Prager [24].

As this partition is random, they predict that at low $q$, one sees the structure factor of - say - a water domain (in the oil rich side), and the intensities approximately follow a Guinier law (the « equivalent radius of gyration $R_{\mathrm{G}}$ " is equal to $0.572 c^{-1 / 3}$, where $c^{-1 / 3}$ is the characteristic length of the microemulsion related to the specific surface in the microemulsion $c_{\mathrm{S}} \Sigma$ by : [9] $c_{\mathrm{S}} \Sigma=5.82 c^{1 / 3} \phi_{0} \phi_{\mathrm{w}}$, thus $c^{-1 / 3}=\frac{5.82 \phi_{0} \phi_{\mathrm{w}}}{c_{\mathrm{S}} \Sigma}=$ $0.97 \xi_{k}$; within this model $R_{\mathrm{G}}=0.55 \xi_{k}$ ).

This prediction does not agree with the experimental results at very low $q$ (the intensities exhibit a peak). But again, we note a remarkable coincidence : the experimental relation found in the inversion zone between $L=3.8 q_{1}^{-1}$ and $\xi_{k}\left(L=0.6 \xi_{k}\right)$ is very close of the Kaler-Prager theoretical prediction $R_{\mathrm{G}}=0.55 \xi_{k}$.

The peak existence is puzzling within the frame of the random models, however it is not by itself a proof in favour of a model of - say - hard spheres. It is mainly a proof in favour of a model less random than that of Kaler and Prager.

Considering the results of the preceding section, it is worthwhile to try to understand how a peak can arise within the frame of the random models :

Probably, the correlations between neighbouring oil and water domains linked by the interfacial film are not negligible as was supposed by Kaler and Prager. The situation could be analogous to that observed in hydrogenated and deuterated diblock copolymer melts : the double constraint, global incompressibility and link between the two distinct parts of a polymer chain, gives a correlation peak in the neutron scattering spectra [25]. 
At $\phi_{0}=0.5$, the experimental Bragg spacing $d^{*}$ is almost equal to $2 \xi_{k}$ (at $\phi_{0}=0.5 q_{1}^{-1}\left(\phi_{0}=0.5\right)=$ $\left.40 \AA, \xi_{k}=6.5 q_{1}^{-1}=260 \AA\right)$. This is consistent with a picture of domains in close contact. Let us consider this symmetric case $\phi_{0}=0.5$ and assume that the polydispersity of the domain sizes is very small (this is at variance with the Kaler-Prager model, but in accordance with the picture of a rigid film at scale smaller than $\left.\xi_{k}[8]\right)$. If one cuts the medium by a straight line, the water sections (where the electron density fluctuation $\eta(x)$ is positive) alternate with the oil section (where $\eta(x)$ is negative); if the size polydispersity is small, these sections have approximately the same size, the electron density fluctuation $\eta(x)$ along the line is an almost periodical fonction. The autocorrelation fonction $\gamma(x)$ of $\eta(x)$

$$
\left(\gamma(x)=\int \mathrm{d} y \eta(x+y) \eta(y)\right)
$$

is positive at small distances and becomes negative and eventually oscillates (if $\eta(x)$ is truly periodical, $\gamma(x)$ also is periodical), if the negative part of $\gamma(x)$ is sufficiently large, this can lead naturally, by Fourier transform, to a peak in the scattered intensity.

ii) In the B samples outside the inversion zone, the deviations from a Guinier law are possibly due to attractive Van der Waals interactions between droplets or to the existence of a critical point in this phase diagram region [26], or also, to the presence of large aggregates. These possibilities may be interrelated in surfactant systems [27a] and in microemulsions [27b]. In the $q$-range studied, we lack information to discriminate between them and to explain the apparent similarity of the intensity plots in reduced units (Fig. 7 samples $\phi_{0}=0.9$ and 0.8 at different $c_{\mathrm{s}}$ ).

iii) In the A samples, $S=3$ and $4 \%$, the interpretation is quite different and more straightforward. Repulsive interactions exist because the charge of the water in oil droplets are less screened than at higher salinities. It is also possible that - far from the optimal salinity - the spontaneous curvature of the interfacial film is important, that the film is a spherical indeformable shell and that the droplets are hard spheres. Both effects can lead to a repulsive type peak in the spectra.

We have noted that the Bragg spacing $d^{*}$ associated to the peaks are approximatively equal to 4 times the radius : $\left(d^{*}(3 \%)=250 \AA ; R(3 \%)=4.6 q_{1}^{-1}=63 \AA\right.$; $\left.d^{*}(4 \%)=330 \AA ; R(4 \%)=80 \AA\right)$.

\section{Conclusion.}

Among the two possible kinds of concentrated microemulsions :

i) concentrated oil or water spheres dispersions,

ii) bicontinuous random oil-water dispersions,

the Winsor phases in the vicinity of the three phases region that we studied are seemingly of the second type at high water and oil concentration. The existence of this type of microemulsion - which also contains a very small quantity of surfactant - critically depends on the chemical parameters, salinity and surfactant amount. An essential condition seems to be that the spontaneous curvature of the interfacial film is weak (or in other terms, that the surfactant is as much hydrophile as lipophile).

Although detailed models are still lacking, the $\mathrm{X}$-ray experiments are a useful means for studying the structure of these systems. They confirm the existence of an interfacial film in microemulsion. They allow the surface per surfactant polar heads and the characteristic size of the microemulsions to be measured.

The structure of these Winsor microemulsions is well described by the Talmon Prager or de Gennes models at the scale of the oil and water domains. It depends only on the oil and water uptakes and on the surfactant concentration, but not on the phase behaviour, nor on the vicinity of a critical point.

The large scale structure and correlations which are observed in the inversion zone are however not explained by the Kaler-Prager model, this could then indicate that, when the oil and water proportions are almost equal, the size of the oil and water domains does not fluctuate very much.

\section{Acknowledgments.}

We thank P. G. de Gennes and G. Jannink for the numerous ideas exchanges and discussions we had; V. Luzzati for hospitality at the "Centre de Génétique Moléculaire "; A. Tardieu and C. Williams for their precious help and advices during the SAXS experiments.

\section{Appendix I.}

ASYMPTOTIC BEHAVIOUR OF SCATTERED INTENSITY at SMall angles. Deviations from Porod's laW. In electronic units, the intensity scattered by an isotropic medium is given in term of the fluctuation $\eta(r)$ of the electron density by :

$$
i(q)=4 \pi V\left\langle\eta^{2}\right\rangle \int_{0}^{\infty} \gamma(r) r^{2} \frac{\sin q r}{q r} \mathrm{~d} r
$$

with

$$
\gamma(r)\left\langle\eta^{2}\right\rangle=\langle\eta(0) \eta(r)\rangle
$$

(cf. (2)).

Often, in inhomogeneous materials or colloidal solutions one can distinguish two phases separated by a sharp interface (holes and matrix of a porous medium, colloidal particules and solvent). Assuming a constant electron density in each phase, Porod and Debye derived the asymptotic form of the scattered intensity :

$$
\lim _{q \rightarrow \infty} \frac{q^{4} i(q)}{\int_{0}^{\infty} q^{2} i(q) \mathrm{d} q}=\frac{1}{l_{\mathrm{p}}}=\frac{A}{4 \phi(1-\phi) V}
$$


$l_{\mathrm{p}}$ can be called Porod's length, $A / V$ is the area of interface per unit volume and $\phi$ is the volume fraction of one of the two phases.

1) Interfacial effects. - In some cases, the interface is not sharp, moreover there can be, as in our case, a substantial contribution to the scattered intensity due to a heavy substance located at the interface. This leads to deviations from Porod's law [28].

For the needs of our analysis, we rederive them by a method suggested by de Gennes.

Let $d$ be the thickness of the interface, along the normal $\mathrm{O} z$ to the interface. The electron density is decomposed into two terms : a step function $\theta$ giving the discontinuity of density and a residual function $\rho(z)$ vanishing outside the interfacial region (Fig. A1).

If $q$, the scattering vector, is smaller than a typical curvature of the interface, but larger than $d^{-1}$, we can only consider the scattering by the interfacial region and particularly the contribution of a rectangular zone of length $L_{x}$ and width $L_{y}$. $(\mathrm{O} x$ and $\mathrm{O} y$ are two axes in the plane of the interfaces, $\mathrm{O} z$ is the normal, the origine $O$ is on the discontinuity of $\theta, n_{1}$ is the electron density for $z<0$ and $n_{3}$ for $z>0$ ).

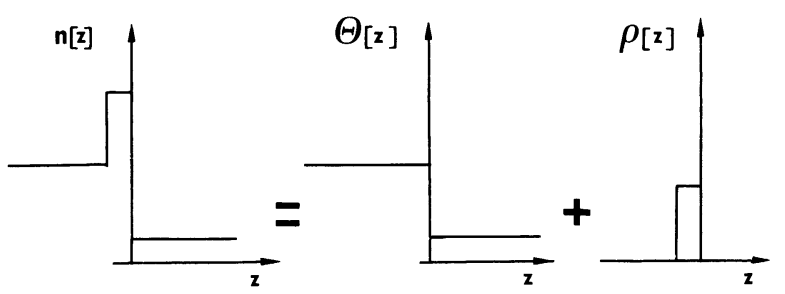

Fig. A1. - Decomposition of the electron density profile through the interface in a step function $\theta$ and a residual function $\rho$.

The amplitude scattered by the rectangle is :

$$
\begin{aligned}
& \mathcal{A}(\mathbf{q})=L_{x} L_{y} \frac{\sin \left(q_{x} L_{x} / 2\right)}{\left(q_{x} L_{x} / 2\right)} \frac{\sin \left(q_{y} L_{y} / 2\right)}{q_{y} L_{y} / 2}\left[\left(n_{1}-n_{3}\right) \frac{1}{i q_{z}}+\tilde{\rho}\left(q_{z}\right)\right] \\
& \tilde{\rho}\left(q_{z}\right)=\int_{-\infty}^{+\infty} \rho(z) \mathrm{e}^{i q_{z} z} \mathrm{~d} z .
\end{aligned}
$$

The intensity is :

$$
|\mathcal{A}(\mathbf{q})|^{2}=\left(L_{x} L_{y}\right)^{2} \operatorname{sinc}^{2} \frac{q_{x} L_{x}}{2} \operatorname{sinc}^{2} \frac{q_{y} L_{y}}{2}\left[\left(n_{1}-n_{3}\right)^{2} \frac{1}{q_{z}^{2}}+\left|\rho\left(q_{z}\right)\right|^{2}-\frac{2\left(n_{1}-n_{3}\right)}{q_{z}} \operatorname{Im} \tilde{\rho}\left(q_{z}\right)\right] .
$$

Now, assuming isotropy, we take the average on the possible orientation of the rectangle. For large $q$ $(q L \gg 1)$, the sinc term will vanish except if $\mathbf{q}$ is normal to the interface; by taking the average sinc $q_{x} L_{x} / 2$ is equivalent to $\left(2 \pi / q_{x} L_{x}\right) . \delta\left(q_{x}\right), \delta$ is the Dirac fonction.

Finally :

$$
\left\langle|\mathcal{A}(q)|^{2}\right\rangle=\frac{2 \pi L_{x} L_{y}}{q^{4}}\left[\left(n_{1}-n_{3}\right)^{2}+q^{2}|\tilde{\rho}(q)|^{2}-2\left(n_{1}-n_{3}\right) q \operatorname{Im} \tilde{\rho}(q)\right] .
$$

The contribution of different portions of the interface (of size $q^{-1}$ ) are incoherent, then additive. The total scattered intensity is :

$$
i(q)=\frac{2 \pi A}{q^{4}}\left[\left(n_{1}-n_{3}\right)^{2}+q^{2}|\tilde{\rho}(q)|^{2}-2\left(n_{1}-n_{3}\right) q \operatorname{Im} \tilde{\rho}(q)\right]
$$

$A$ is the total area of the interface.

For $q d<1$, this is expressed in a more useful form by using the moments of the distribution $\rho$ : $M_{n}=\int_{-\infty}^{+\infty} z^{n} \rho(z) \mathrm{d} z$

$$
q^{4} i(q)=2 \pi A\left\{\left(n_{1}-n_{3}\right)^{2}+q^{2}\left[M_{0}^{2}-2\left(n_{1}-n_{3}\right) M_{1}\right]+q^{4}\left[M_{1}^{2}-M_{0} M_{2}+\frac{1}{3}\left(n_{1}-n_{3}\right) M_{3}\right]+0\left(q^{6}\right)\right\}
$$

The best way to evidence the first deviation from Porod's law is to plot $q^{4} i(q) v s . q^{2}$.

With the electron density profile of figure 5

$$
q^{4} i(q)=2 \pi A\left\{\left(n_{1}-n_{3}\right)^{2}+\left(n_{2}-n_{1}\right)\left(n_{2}-n_{3}\right)(q d)^{2}+0(q d)^{4}\right\} .
$$




\section{Appendix II.}

ThE ELECTRON DENSITY DISTRIBUTION. - The electron density profile through the interfacial film is drawn on figure A2.

We suppose that the oil-water interface is infinitely thin, and that one can distinguish two well defined parts in the film :

- an aliphatic part (width $d^{\prime}$, electron density $n_{\mathrm{A}}$ ) which includes the $\left(\mathrm{CH}_{2}\right) \mathrm{CH}_{3}$ tails of $\mathrm{SdS}$ molecules and some toluene,

- a polar part (width $d$, electron density $n_{\mathrm{p}}$ ) which includes the polar heads : $-\mathrm{OSO}_{3} \mathrm{Na}$ and some brine.

We neglect the influence of butanol on the electronic contrast.

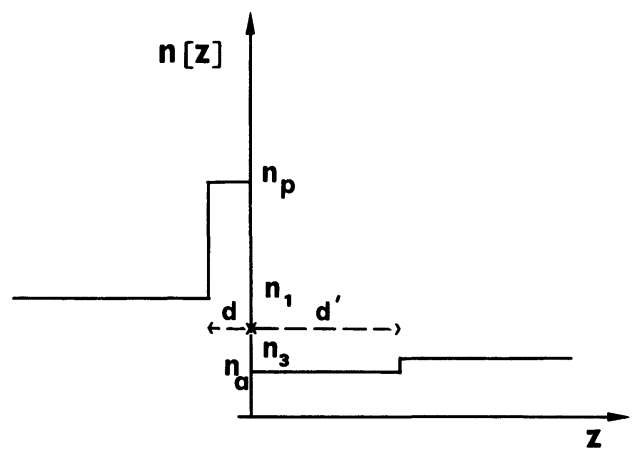

Fig. A2. - Model of electron density profile through the interfacial film.

Table A gives molecular weight, mass density and electron density of all chemical components of the microemulsions. $n_{1}$ is the electron density of brine, $n_{3}$ is the electron density of oil. One has :

$n_{1}=0.334+0.00134 S\left(\AA^{-3}\right)$, where $S$ is the weight (in g) of $\mathrm{NaCl}$ in $100 \mathrm{~g}$ of brine.

$$
=0.343 \AA^{-3} \quad(S=6.5) \text {. }
$$

Let $A$ be the total area of the oil water interface, and $N_{\mathrm{S}}$ the total number of SdS molecules, assumed to be only located at the oil water interface.

$$
A=N_{\mathrm{S}} \Sigma, \quad \Sigma \text { is the surface per polar head. }
$$

The volume of the polar part is $N_{\mathrm{S}} \Sigma d$, evaluating the volume fraction occupied by the polar heads in this part of the film :

$$
\phi_{\mathrm{p}}=\frac{67.8}{\Sigma d}, \quad\left(\Sigma \text { in } \AA^{2}, d \text { in } \AA\right)
$$

$67.8 \AA$ is the molecular volume of $\mathrm{NaOSO}_{3}$ estimated from the density and molecular weight (Table A).

Table A. $-M$ : molecular weight $(\mathrm{g}), d$ : weight den$\operatorname{sity}\left(\mathrm{g} / \mathrm{cm}^{3}\right), n$ : electron density $\left(\AA^{-3}\right)$.

\begin{tabular}{|l|c|c|c|}
\hline & $\mathrm{n}$ & $\mathrm{d}$ & $\mathrm{M}$ \\
$\mathrm{H}_{2} \mathrm{O}$ & 0.334 & 1 & 18 \\
$\mathrm{C}_{7} \mathrm{H}_{8}$ & 0.283 & 0.867 & 92.1 \\
$\mathrm{C}_{6} \mathrm{H}_{12}$ & 0.267 & 0.779 & 84.2 \\
$\mathrm{NaCl}^{\prime} \mathrm{OSO}_{3} \mathrm{Na}$ & 0.870 & 2.915 & 119 \\
$\left(\mathrm{CH}_{2}\right)_{11} \mathrm{CH}_{3}$ & 0.275 & 0.79 & 169 \\
\hline
\end{tabular}

One finds :

$$
n_{\mathrm{p}}-n_{1}=\left(n_{\mathrm{NaSO}_{4}}-n_{1}\right) \phi_{\mathrm{p}}
$$

For $S=6.5$

$$
n_{\mathrm{p}}-n_{1}=\frac{35.7}{\Sigma d} \AA
$$

with toluene,

$$
\begin{aligned}
& n_{1}-n_{3}=0.06 \\
& n_{\mathrm{p}}-n_{3}=0.06+\frac{35.7}{\Sigma d} .
\end{aligned}
$$

Similarly :

$$
n_{\mathrm{A}}-n_{3}=-\frac{2.8}{\Sigma d^{\prime}} \AA^{-3}
$$

$n_{\mathrm{A}}-n_{3}$ is very small, compared to $n_{\mathrm{p}}-n_{1}$ and $n_{1}-n_{3}$ : we have neglected it in the text.

From the measure of $l^{2}=d^{2} \frac{\left(n_{\mathrm{p}}-n_{1}\right)\left(n_{\mathrm{p}}-n_{3}\right)}{\left(n_{1}-n_{3}\right)^{2}}$, one can estimate $\Sigma_{i}$ for $S=6.5$ (and toluene)

$$
l^{2}=\left(\frac{595}{\Sigma}\right)^{2}\left(1+d /\left(\frac{595}{\Sigma}\right)\right) \quad(l \text { in } \AA)
$$

with cyclohexane and brine $(S=5.5 \%)$

$$
l^{2}=\left(\frac{470}{\Sigma}\right)^{2}\left(1+d /\left(\frac{470}{\Sigma}\right)\right) \quad(l \text { in } \AA) .
$$




\section{References}

[1] PrInCE, L. M. (ed), Microemulsions, theory and practice (Academic Press) 1977.

[2] Winsor, P. A., Solvent properties of amphiphilic compounds (Butterworths, London) 1954.

[3] Own observation. Also Roux, D., Belloce, A. M., Leblanc, M. S., Chem. Phys. Letters 94 (1983) 156.

[4] Agterof, W. G. M., van Zomeren, J. A. J. and VriJ, A., Chem. Phys. Lett. 43 (1976) 363.

Cebula, D. J., Ottewil, R. H., Ralston, J., Pusey, P. N., J. Chem. Soc. Faraday Trans. I 77 (1981) 2585.

Cebula, D. J., Myers, O. Y., Ottewil, R. H., Coll. Polym. Sci. 260 (1982) 96.

Lemaire, B., Bothorel, P., Roux, D., J. Phys. Chem. 87 (1983) 1023.

Brunetti, S., Roux, D., Belloce, A. M., Fourche G. Bothorel, P., J. Phys. Chem. 87 (1983) 1028.

Cazabat, A. M., Langevin, D., Pouchelon, A., J. Coll. Interf. Sci. 73 (1980) 1.

Cazabat, A. M., Langevin, D., J. Chem. Phys. 74 (1981) 3148.

[5] Dvolaitzsky, M., Lagües, M., Lepesant, J. P., Ober, R., Sauterey, C. and Taupin, C., J. Phys. Chem. 84 (1980) 1532.

[6] Ober, R., Taupin, C., J. Phys. Chem. 84 (1980) 2418.

[7] SCriven, L. E., in Micellization, Solubilization and Microemulsions, ed. by K. Mittal (Plenum Press) 1977, vol. 2, p. 877.

[8] De Gennes, P. G., Taupin, C., J. Phys. Chem. 86 (1982) 2294.

[9] Talmon, Y., Prager, S., J. Chem. Phys. 69 (1978) 2984.

[10] De Gennes, P. G., Jouffroy, J., Levinson, P., J. Physique 43 (1982) 1241.

[11] Surface phenomena in enhanced oil recovery ed. by D. O. Shah (Plenum Press) 1981

[12] Safran, S. A., Turkevitch, L. A., Phys. Rev. Lett. 50 (1983) 1930.

[13] The phase diagram of the system brine, toluene, SdS, butanol is extensively studied for different salinities in :

Belloce, A. M., Biais, J., Clin, B., Lalanne, P., Lemanceau, B., J. Coll. Interf. Sci. 70 (1979) 524.

[14] Pouchelon, A., Meunier, J., Langevin, D., Cazabat, A. M., J. Physique Lett. 41 (1980) L-239.
[15] Pouchelon, A., Thèse de Docteur Ingénieur - Université Paris VI (1982).

Cazabat, A. M., Langevin, D., Meunier, J., PoucheLON, A., Adv. Coll. Int. Sci. 16 (1982) 175.

The proportion of the system studied in this thesis are slightly different of those of Ref. [14], which we have chosen.

[16] Gabriel, A., Dupont, Y., Rev. Sci. Inst. 43 (1972) 1600.

[17] The description of the two X-ray set-up may be found in : VAchette P., Thèse de Doctorat d'Etat Université Louis Pasteur, Strasbourg (1979).

[18] Bennett, K. E., Hatfield, J. C., Davies, H. T., Macosko, C. W. and Scriven, L. E., in Microemulsions ed. by I. D. Robb (Plenum Press) 1982, p. 65.

[19] LANDAUER, R., in Electric transport and optical properties of inhomogeneous media, Ohio State Univ. (1977), eds J. C. Garland and D. B. Tanner (Amer. Inst. of Physics) 1978, p. 1.

[20] GuInIER, A., Fournet, G., Small-angle scattering of $X$-rays (J. Wiley) 1955.

[21] Glatter, O., KRatKy, O., Small-angle X-rays scattering (Academic Press) 1982.

[22] Porod, G., Koll. Z. 124 (1951) 83.

[23] A peak was also observed by neutron scattering in the middle phases of the Winsor system of Ref. [15].

Drifford, M., TAbony, J., DE Geyer, A., Communication at Colloque de la Société de Chimie Physique : Micelles et Microémulsions : structure et réactivité. C.E.N. Saclay. Orme des Merisiers. 24-25 mai 1983 (unpublished).

[24] Käler, E. W., Prager, S., J. Coll. Int. Sci 86 (1982) 359.

[25] De Gennes, P. G., Scaling Concepts in Polymer Physics (Cornell University Press, Ithaca) 1979.

[26] Cazabat, A. M., Langevin, D., Meunier, J., PouCHELON, A., J. Physique Lett. 43 (1982) L-89.

[27] a) Triolo, R., MAGID, L. J., Johnson, J. S. Jr., Child, H. R., J. Phys. Chem. 86 (1982) 3689.

b) Cazabat, A. M., Chatenay, D., Langevin, D., Pouchelon, A., J. Physique Lett. 41 (1980) L-441.

[28] Ruland, W., J. Appl. Cryst. 4 (1971) 70. 